LBL- -30701

DE91 013750

\title{
PARASITIC CROSSING AT AN ASYMMETRIC B FACTORY, APLARY*
}

\author{
Y.H. Chin \\ Lawrence Berkeley Laboratory \\ University of California \\ Berkeley, CA 94720
}

* This work was supported by the Director, Office of Energy Research, Office of High Energy and Nuclear Physics, High Energy Physics Division, of the U.S. Department of Energy under Contract No. DE-AC03-76SF00098.

\section{MASTER}




\title{
PARASITIC CROSSING AT AN ASYMMETRIC B FACTORY, APIARY ${ }^{*}$
}

\author{
Y.H. Chin \\ Accelerator \& Fusion Research Division \\ Lawrence Berkeley Laboratory \\ Berkeley, CA 94720
}

\section{Abstract}

Errects of parasitic crossings ("near miss" collisions of two counter-rotating beams at unwanted positions near the IP) are studied in terms of computer simulations for an asymmetric B Factory, APIARY-6.3d. Beams are separated horizontally at the first parasitic crossing points by about 7.6 times the horizontal rms size of the low energy beam (the larger in size of the two beams), $\sigma_{0 x_{1}+.}$ Simulations, including both the beam collision at the IP and parasitic crossings, have been performed for different separation distances, $d$. It is found that the ratio $d / \sigma_{0 x}+$ is a good scaling parameter of beam blowup behavior. The results show that beam blowup due to the parasitic crossings is diminished for. $d \geq 7 \sigma_{0 x_{1}+}$, in agreement with the bunch separation experiment ar CESR. Thus, the nominal separation $7.6 \sigma_{0 x_{1}+}$ tums out to be acceptable, but with only a small margin. Some methods to mitigate the effects of the parasitic crossings are discussed.

\section{INTRODUCTION}

The attainable luminosity in an asymmetric storagering collider for a B Factory will be determined to 2 large extent by the physics of the beam-beam interaction. Extensive studies of the beam-beam dynamics under asymmetric energy conditions have icen done, and the idea of the so-called "energy transparency symmetry" was suggested to put the two beams on an equal footing as far as transverse dynamics is concemed [1]. Most of those studies consider only primary head-on collision of two beams at the IP. For the APIARY-6.3d, the bunch spacing is only $1.26 \mathrm{~m}$, so that the bunches experiencs iong-range collisions on the way into and out of the IP region (where both beams travel in a common vacuum pipe). These coilisions are called "parasitic crossings." There are six parasitic crossings symmetrically locared on either side of the IP. Of these, the first parasiti: crossing (the one closest to the IP) on either side has the dominant effect on beam dynamics due to the small beam separation and the large vertical beta function. The nominal parameters at the IP and the first parasitic crossing point for the two rings of APIARY-6.3d are listed in Table 1. In this table, $\Delta s$ is the distance between the IP and the first parasitic crossing, $d$ is the separation

* Supported by Director. Oftice of Energy Research, Office of High Energy and Nuclear Phytics, High Energy Physics Division, of the U.S. Department of Energy under Contract No. DE-AC0376SFC0098. distance between the two orbits at the parasitic crossing, $\Delta v$ is the tune advance between the IP and the first parasitic crossing. $\beta$ is the beta function, and $\tau_{0}$ is the rms nominal beam size. Horizontal and vertical qua ities are denoted by the subscripts $x$ and $y$, respectively.

Table 1

APIARY-6.3d nomir 1 parameters at the IP and the Irst pa-asitic crossing

\begin{tabular}{|c|c|c|c|c|}
\hline \multirow[b]{2}{*}{$\begin{array}{l}\Delta s(\mathrm{n}) \\
d(\mathrm{~mm})\end{array}$} & \multicolumn{2}{|c|}{$\begin{array}{c}\text { Low Energy Ring } \\
\left(L E R, e^{+}\right)\end{array}$} & \multicolumn{2}{|c|}{$\begin{array}{c}\text { High Energy Ring } \\
\text { (HER, } \odot)\end{array}$} \\
\hline & \multicolumn{4}{|c|}{$\begin{array}{l}0.63 \\
-82\end{array}$} \\
\hline & IP & 1st PC & IP & 1st $P C$ \\
\hline$\Delta v_{x}$ & 0 & 0.1643 & 0 & 0.1111 \\
\hline$\Delta v_{y}$ & 0 & 0.2462 & 0 & 0.2424 \\
\hline$\beta_{x}(m)$ & 0.375 & 1.51 & 0.75 & 1.30 \\
\hline$\beta_{y}(\mathrm{~m})$ & 0.015 & 25.23 & 0.03 & 13.01 \\
\hline$\omega_{x}(\mu \mathrm{m})$ & 186 & 373 & 186 & 245 \\
\hline$\omega_{0 y}(\mu \mathrm{m})$ & 7.4 & 302 & 7.4 & 153 \\
\hline$d / \sigma_{20}$ & - & 7.6 & - & 11.5 \\
\hline
\end{tabular}

Parasitic crossings have the potential to induce significant blowup in the vertical beam size of the low energy ring (LER), because they excite codd-order resonances and because the vertical long-range tune shift of the LER is as large as the head-on tune shift at the $\mathbb{P}$. Obviously, if the separation is large enough, effects of the parasitic crossings diminish. We carry out simulations to see if the present nominal separation $d=7.6 \sigma_{0 x}+$ gives acceptable performance. A more detailed description of the present study can be found in Ref. 2.

\section{SIMULATION TECHNIQUE}

Once the two rings are filled with bunches, pairs of collisions at the IP are fixed; that is, each bunch of one ring collides only with the same partner in the other ring. Therefore, the beam-beam dynamics can be simulated with one bunch per ring. However, when parasitic crossings are included, all the bunches can "talk" to each other directly or indirectly. A completely faithful simulation for APIARY6.3d would require 1658 bunches per ring, pushing the CPU time beyond practical limits. If the coherent beam-beam oscillation does not play an imporant role in beam blowup, the "talk" between bunches may not need to be simulated exactly. At the same time, the particle distributions do not 
differ much from bunch to bunch. Under these assumptions, we may adopt the following technique to allow us to use only one bunch per ring. Two bunches are counter-rotating in the two rings (see Fig. 1). yntien the $e-$ bunch is at the parasitic crossing PC+, the $e^{+}$bunch is at the other parasitic crossing. PC-. To calculate the beam-beam force on the $e^{-}$bunch from the $e^{+}$bunch at $P C+$, we use the particle distribution of the $e^{+}$ bunch at PC-. The same technique is applied to the $e^{+}$bunch.

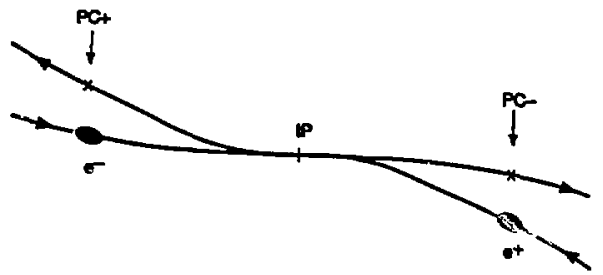

Figure 1. Shematic layout of the interaction region.

\section{SDMULATION RESULTS}

The main parameters of APIARY-6.3d used in the simulations are listed in Table 2.

Table 2

Main parameters of APLARY-6.3d

\begin{tabular}{|l|c|c|}
\hline & LER $\left(e^{+}\right)$ & HER $\left(e^{-}\right)$ \\
\hline Energy, $E(\mathrm{GeV})$ & 3.1 & 9 \\
\hline Circumfererce, $C(\mathrm{~m})$ & 2200 & 2200 \\
\hline $\begin{array}{c}\text { Nominal emittance, } \\
\varepsilon_{x x} \text { (nm-rad) } \\
\varepsilon_{0 y} \text { (nm-rad; }\end{array}$ & 92 & 46 \\
\hline Bunch length, $\sigma_{s}(\mathrm{~cm})$ & 3.6 & 1.8 \\
\hline $\begin{array}{l}\text { Damping time, } \\
\tau_{x}=\tau_{y} \text { (turns) }\end{array}$ & 1.0 & 1.0 \\
\hline Bunch current, $I_{b}(\mathrm{~mA})$ & 4400 & 5014 \\
\hline Synchrotron tune, $Q_{s}$ & 0.0403 & 0.0520 \\
\hline $\begin{array}{l}\text { Nominal beam-beam } \\
\text { tune shift, } \xi_{0 x}=\xi_{0 y}\end{array}$ & 0.03 & 0.03 \\
\hline
\end{tabular}

We have selected the fractional tunes of the working point to be $v_{x}=0.09$ and $v_{y}=0.05$ for both beams at the present time; a thorough tune scan remains to be carried out for actual operation. For these parameters, the primary simulation result without parasitic crossings shows $23 \%$ beam blowup in the vertical size in the LER. The other three beam sizes remain practically unchanged from their nominal values. Figure 2 shows the beam blowup factor as a function of the separation $d / \sigma_{0 x}+$ where all other parameters are kept fixed. The corresponding luminosity as a function of $d / \sigma_{0 x},+$ is shown in Fig. 3. From Fig. 2, it can be sien that the separation $d=$ $7 \sigma 0 x_{\text {, }}+$ should be enough to consider that the effects of the parasitic crossings are negligible. Accordingly, the luminosity is only reduced by about $10 \%$ from its design value. This result agrees with the separation experiment at CESR [3] that concludes that at least 6 "effective" $\sigma_{0 x}$ (practically, $6+$ $1 \sigma_{0 x}$ ) separation is required to maintain a one-hour beam lifetime.

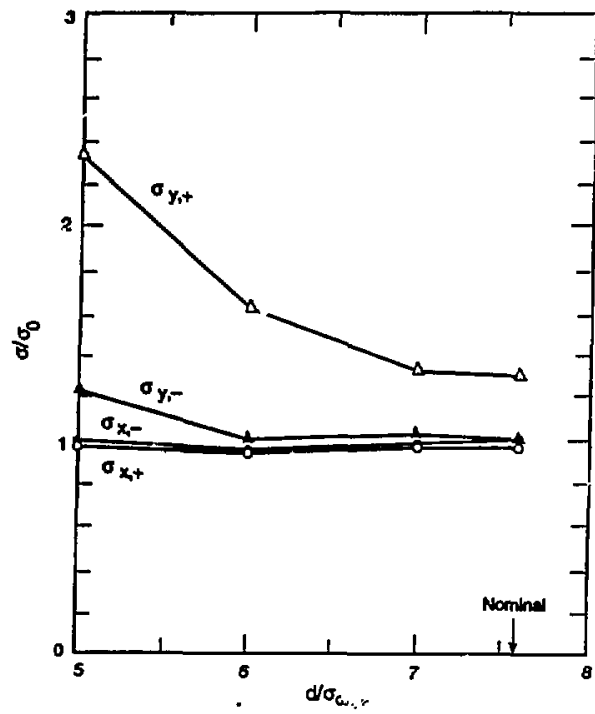

Figure 2. RMS beam sizes as a function of the relative separation $d / \sigma_{0 x_{1}+}$ for the nominal APLARY6.3d parameters. The subscripts label HER $(-)$ and LER (+). The nominal team separation at the parasitic crossing is indicated by the arrow.

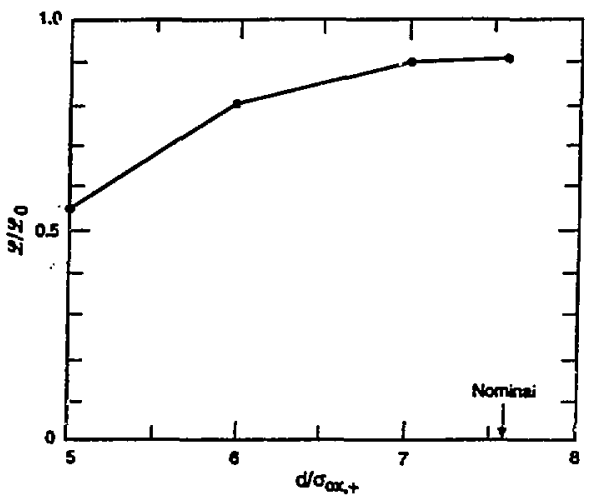

Figure 3. Luminosity as a function of $d / \sigma_{0 x,+,}$ for the nominul APIARY-6.3d parameters.

Although the nominal separation $d=7.6 \sigma_{0 x}+$ turns out to be large enough, the safety margin for closed-orbit 
distortion and so forth is not that great. A simple solution for this would be to increase the separation distance, and that approach is now under study. Here, however, we intend to explore ather possibilities in order to improve luminosity, particulariy for a large value of $\xi_{0}$. The first such possibility is to increase the beta function at the IP, $\beta^{*}$, of the LER. The idea is to make the tune modulation due to the synchrotron motion at the IP closer to the energy transparency condition, and simultaneously to reduce the beta function of the LER at the parasitic crossing. Therefore, the beam size at the parasitic crossing is also reduced and the relative separation $d / \sigma_{0 x_{0}+}$ increases. The penalty is a large bw-energy beam current. $A$ preliminary simulation result without parasitic crossings shows that the two, beams blow up more symmetrically and the luminosity gets closer to its nominal value.

Another possibility is to increase the bunch spacing from $2 \lambda_{r f}=1.26 \mathrm{~m}$ to $3 \lambda_{r f}=1.89 \mathrm{~m}$ by filling the rings with bunches every third if bucket, instead of every second bucketh while other lattice parameters are kept fixed. Here, $\lambda_{\text {,f }}$ is the If wave length. This pushes the parasitic crossing farther away from the $\mathbb{P}$ and the separation distance becomes larger. Now, the separation $d$ at the first parasitic crossing increases from $2.82 \mathrm{~mm}$ to a considerably large value of $7.41 \mathrm{~mm}$. The beta function at the parasitic crossing increases also, so that the relative separation $d / \sigma_{0 x_{1}+}$ increases from 7.6 to 9.2 . In order to maintain the huminal $\xi_{0}$ and keep the luminosity constant. the bunch current and the emittance also must increase by $50 \%$. These changes are still acceptable in terms of beam instability thresholds and dynamic aperture considerations. The simulation resules are shown in Figs. 4 and 5. We can see practically no beam blowup at the nominal separation, and the luminosity is close to the nominal value. The safety margin of $d$ is now sufficiently greater than the previous case (note that the beam size at the parasitic crossing increases by a factor of about 2). Good luminosity performance remains even for a larger $\xi_{0}=0.05$.

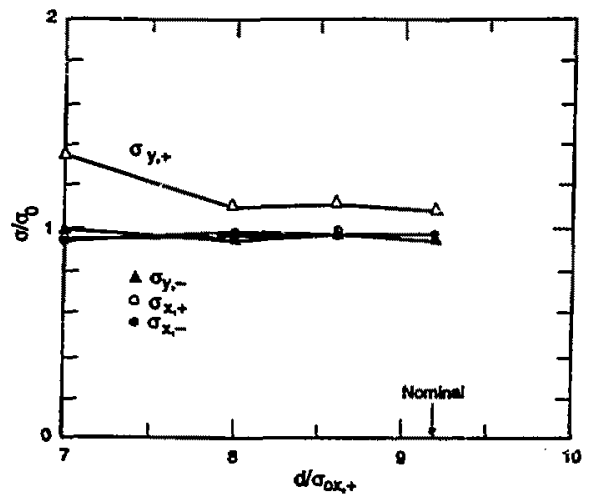

Figure 4. RMS beam sizes as a function of $d / \sigma_{0 x,-r}$ for APIARY-6.3d, in the case where bunches are filled every third bucket instead of every second bucket.

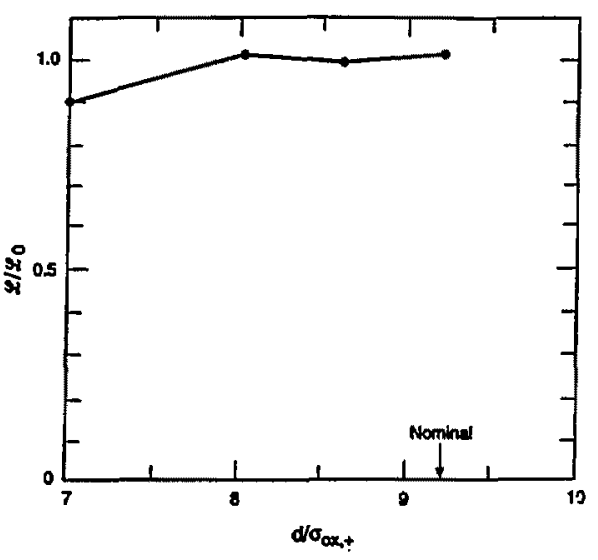

Figure 5. Luminosity as a function of $d / \sigma_{0 x,+}$ for APIARY-6.3d, corresponding to Fig. 4.

\section{CONCLUSIONS}

Simulation results including the effect of parasitic crossings for APIARY-6.3d show that the nominal separation is large enough that heam blowup due to the parasitic crossings disappears and the luminosity reduction is only $10 \%$ from its nominal value for $\xi_{0}=0.03$. However, the safcty margin in terms of separation tolerance is low. To mitigate the effects of the parasitic crossings, one such possibility is to change the lattice parameters, such as the beta functions at the IP and at the parasitic crossing, so that the relative separation $d / \sigma_{0 x}$ increases. Another possibility is to increase the bunch spacing from $2 \lambda_{r f}=1.26 \mathrm{~m}$ to $3 \lambda_{r f}=1.89 \mathrm{~m}$ by filling the rings with bunches every thind bucket rather than every second bucket. In this way, the optics parameters can be kept fixed. Preliminary simulation results for the case of filling every third bucket show improvement in the beam sizes and the luminosity.

The author would like to thank M. Zisman for helpful discussions and proofreading of the manuscript.

\section{REFERENCES}

[1] Y.H. Chin in Beam Dynamics Issues of High Luminosity Asymmetric Collider Rings, AIP Conf. Proc. 214, edited by A.M. Sessler, Berkeley, CA (1990), pp. 424-433.

[2] An Asymmetric B Factory Based on PEP, Conceptual Design Report, Lawrence Berkeley Laboratory Report LBL PUB-5303, Febnuary, 1991.

[3] D.H. Rice in Beam Dynamics Issues of High Luminosity Asymmetric Collider Rings, AIP Conf. Proc. 214, edited by A.M. Sessler, Berkeley, CA (1990), pp. 219-234. 